\title{
Le sari à l'européenne. Vêtement et militantisme en Inde coloniale
}

Europeanized sari. Dress and militancy in colonial India

\section{Arundhati Virmani}

\section{(2) OpenEdition}

1 Journals

\section{Édition électronique}

URL : http://journals.openedition.org/clio/10771

DOI : 10.4000/clio. 10771

ISSN : 1777-5299

Éditeur

Belin

\section{Édition imprimée}

Date de publication : 31 décembre 2012

Pagination : 129-152

ISSN : 1252-7017

\section{Référence électronique}

Arundhati Virmani, «Le sari à l'européenne. Vêtement et militantisme en Inde coloniale », Clio.

Femmes, Genre, Histoire [En ligne], 36 | 2012, mis en ligne le 31 décembre 2014, consulté le 01 mai 2019. URL : http://journals.openedition.org/clio/10771; DOI : 10.4000/clio.10771

Tous droits réservés 


\title{
Le sari à l'européenne. Vêtement et militantisme en Inde coloniale
}

\author{
Arundhati VIRMANI
}

J'en suis arrivé à la conclusion que les gens résistent à changer vêtements, coiffure et barbe... bien plus que leurs conditions politiques ou économiques... Il n'y a pas de doute que les vêtements ont exercé une influence puissante sur l'esprit. Quelle grande influence ont-ils pu avoir sur l'histoire ?1

Le stéréotype dominant présente les tenues vestimentaires des Anglaises en Inde comme de pâles imitations des modes et styles métropolitains, dépendantes des modèles reçus d'Angleterre pour maintenir les standards de l'élégance. Cette impression est renforcée dans leurs correspondances. Le vêtement féminin semble être dès lors une extension d'un programme impérial masculin, qui voit les chapeaux et les robes, les collants et les corsets, comme autant de symboles à préserver au sein du système impérial et de son univers de grandeur et de rituels grandioses : un élément de continuité essentiel en des temps incertains ${ }^{2}$. Mais le système de l'esthétique féminine, au carrefour de la culture matérielle, du genre et du symbolisme politique, fonctionne comme un lieu pour la mise en scène de l'identité, l'exercice, et la transgression, du contrôle et de l'autorité. Plus encore dans une situation coloniale, les interactions entre les esthétiques des cultures dominantes et des cultures locales, ou entre les pratiques d'habillement distinctives, ont masqué les négociations

1 Nehru Memorial Museum and Library, New Delhi (NMML), Padmaja Naidu Papers, J. Nehru à Padmaja Naidu, in Gopal \& Iyengar 2003 : 595.

2 Cette perspective domine les analyses pourtant abondantes sur le statut et les rôles de la femme anglaise en Inde. Par exemple Buettner 2004 ; Trollope 1983 ; MacMillan 1988 ; Johnson 2007 ; Shrimpton 1992. 
plus complexes autour de la reconnaissance de la différence. Les choix des vêtements, des matériaux, des couleurs et des accessoires deviennent dans les colonies des stratégies personnelles de représentation, de communication ou de contestation ${ }^{3}$. Pour le gouvernement britannique de l'Inde, le vêtement sert à impressionner ou à dominer les sujets coloniaux et à accentuer la distance entre les gouvernants et les gouvernés. Les situations et les moments précis comme les grandes mises en scène de l'autorité impériale (les «Durbar») mettent en évidence le pouvoir symbolique des habits, encore plus chez les femmes que chez les hommes : lors du Durbar de 1903 à Delhi, la robe de soie de Mary Curzon, épouse du vice-roi Lord Curzon (1899-1905), donne à voir le régime anglais, le Raj, comme une entreprise de séduction par la beauté, capable de mettre en valeur les traditions indiennes : faite par des artisans indiens de fils d'or tissés, elle est couverte de plumes de paon, rehaussées d'une émeraude dans chaque œil ${ }^{4}$. Symboliquement, le corps de la première femme du Raj exhibe ainsi la synthèse impériale de la force (britannique), de la richesse (indienne) et de la séduction (féminine). Cependant, certaines pratiques vestimentaires des femmes anglaises échappent à ces cadres d'analyse. Il ne s'agit pas seulement de cas de transgression ponctuelle ou d'imitation, mais des exemples d'adoption, à la suite d'un re-travail compliqué, de modèles d'habillement des Indiennes.

Les vêtements adoptés par trois femmes britanniques célèbres, Annie Besant, Margaret Noble et Madeleine Slade, qui ne sont ni des robes européennes, ni des saris proprement dits, obligent à reprendre les questions du vêtement féminin dans les colonies sans s'enfermer dans la question des légitimités impériales. Leurs choix vont bien audelà d'une manifestation de leurs préférences esthétiques ou d'un caprice temporaire. Parce qu'elles étaient des femmes participant activement aux mouvements nationalistes et impliquées dans les débats publics sur l'identité et la nationalité indiennes, leur rejet des robes européennes était sans doute une déclaration anti-impériale.

3 Les changements dans les pratiques vestimentaires des femmes colonisées sont étudiés par Karlekar 2006 ; Bhatia 2003.

4 Thomas 2007. 
Mais, tout en questionnant les arrangements domestiques consensuels de l'impérialisme et en s'y opposant, ces choix personnels révèlent des négociations plus complexes entre cultures dominantes et dominées, entreprises par des femmes anglaises et non par des hommes anglais. Quelle était la perception des Indiens, et plus particulièrement des Indiennes, qui luttaient elles-mêmes pour trouver un équilibre entre la tradition et la modernité dans un contexte colonial? Comment peut-on comprendre ces formes d'habillement? À partir de l'élaboration de vêtements pour permettre aux Anglaises d'affronter le climat de l'Inde tout en respectant les codes de l'époque victorienne, cette recherche examine comment certaines femmes peuvent proposer d'autres modèles vestimentaires qui, en créant un espace intermédiaire, contestent aussi bien les modèles britanniques que ceux des élites indiennes. Ces vêtements, porteurs d'une certaine "modernité », ne sont le produit ni d'une indigénisation ni d'un métissage : la subtilité du message, qui renvoie dos à dos dominants et dominés, l’a longtemps rendu invisible.

\section{Une esthétique féminine pour les colonies}

Les modes féminines anglaises en Inde sont fortement soumises aux recommandations de la presse métropolitaine. Journaux, magazines de mode, littérature de voyage, publicités ou livres écrits par des femmes ayant vécu en Inde, offrent des conseils pratiques sur la garde-robe essentielle ainsi que sur la place de la mode ${ }^{5}$. Les Anglaises embarquant pour l'Inde sont ainsi soumises à un véritable flot de conseils pratiques, esthétiques, médicaux, commerciaux concernant aussi bien les vêtements à emporter que les règles et les codes d'habillement et l'importance de se vêtir selon les conventions anglaises dans une société où les Anglais constituent une minoritée. Ce sentiment est renforcé après la révolte de 1857, quand les attaques contre la population civile anglaise, y compris des femmes et des enfants, ont soudé la communauté anglaise par des règles d'apparence et de comportement. En contexte colonial, les Anglaises représentent

5 Ceci depuis le milieu du XIX e siècle: Illustrated London Nens, 25 mars 1843 ; Bradshaw 1884 ; Cassell 1865 ; Diver 1909 ; Lyttleton 1892.

6 Parkes 1850. 
davantage l'empire et symbolisent le pouvoir et l'autorité impériale. Parmi d'autres, l'Englishwoman in India (1909) incite les dames à

apporter avec elles leurs propres patrons (échantillons) de chaque article, allant d'une chemise à une robe avec des dimensions marquées sur chaque pièce, pour qu'ainsi elles puissent acheter ce qui leur plaît en Inde et les faire fabriquer par le couturier indigène dans leur résidence à moindre coût. En laissant leurs mesures à un modiste en Angleterre, elles peuvent recevoir (par colis postal) les patrons en papier aussi bien que les meilleurs magazines de modes à un prix raisonnable. Mais soyez sûre d'apporter toute la garniture, dentelles, boutons et fils, car ces éléments ne peuvent pas se trouver dans le pays ${ }^{7}$.

L'autorité sur ces questions, le manuel de Floral Steele et de Grace Gardiner (1890), conseille aussi à ses lectrices de prendre des robes du soir « vraiment chic » car

les gens s'habillent plus en Inde qu'en Angleterre. Nous ne sommes pas partisanes de l'habillement relâché, au contraire, nous déconseillerons tout abandon à la lassitude et à l'indifférence qui affectent les personnes les plus énergiques dans la chaleur tropicale ${ }^{8}$.

En effet, dès que les femmes britanniques mettent un pied sur le bateau, elles se savent épiées par le regard critique aussi bien des autres femmes que des hommes, tant européens qu'indiens. Bref, leur identité vestimentaire, présente et future, est fixée dès leur départ d'Angleterre, avec des garde-robes emportées pour deux à trois ans. Car, en Inde, la vie sociale anglaise, les clubs, les weekends, les Durbars et les visites officielles nécessitent le maintien d'un certain standard dans l'apparence pour éviter ridicule et dérision, et pire, l'ostracisme de la communauté anglaise. Chaque activité requiert un habit spécifique: équitation, tennis, promenade, chasse, bals ou, tout simplement, prendre un thé...

Les films de l'Inde coloniale montrent les Anglaises en jupes longues, avec chapeaux et ombrelles, comme si elles étaient toujours en métropole. Une photo de la mère du jeune fonctionnaire anglais Malcolm Darling, pendant sa visite en 1907 à son fils alors en poste au Dera Ghazi Khan, près de la frontière afghane, la montre prenant son thé sur l'Indus avec un chapeau impressionnant, incarnant la respectabilité de la matrone victorienne.

Bradshaw 1884

8 Steel \& Gardiner 1890. 
D'autres documents visuels montrant des Anglaises dans leur maison, à la chasse ou accompagnant leur mari dans leurs tournées, confirment l'impression qu'elles ne s'éloignaient pas des modes anglaises. Dans les années 1930, le développement des catalogues et du transport rapide renforce la dépendance des femmes vis-à-vis de la métropole. Les publicités dans des magazines comme Illustrated London, News, Sketch, Tatler, Bystander Sphere, Graphic and Britannia \&o Eve annoncent l'ouverture des services de colis aériens depuis l'Angleterre vers l'Inde, l'Égypte, le Soudan, « en accord avec Imperial Airways Limited $»^{9}$. Et même quand l'usage de la machine à coudre se répand en Inde dans les années 1920 et que le couturier local (darž) devient l'élément essentiel de l'économie domestique, cela ne fait que renforcer le modèle métropolitain imité et reproduit à moindre coût. Les conseils de la littérature de voyage l'expliquent :

C'est un âge de changement; de nouvelles matières s'introduisent continuellement et vous pouvez aussi bien faire venir la description des modèles afin de vous faire une nouvelle robe avec la matière portée en Angleterre peut-être deux ou trois ans plus tôt ${ }^{10}$.

Leur éloignement n'empêche pas les Anglaises de suivre fidèlement les derniers styles, qu'elles réclament par lettre à leur famille restée en métropole. Quelques concessions aux contextes et aux coutumes locales se font avec la conscience qu'elles sont ponctuelles. Même les missionnaires, plus enclins à s'adapter aux milieux locaux, admettent qu'ils le font de façon tout à fait exceptionnelle. Quand Elizabeth Ferrar, qui travaille avec la Mission de Dublin University à Hazaribagh (Bihar) et en Orissa dans le diocèse de Chota Nagpur dans les années 1930, visite les habitants, elle «met un sari» sans pour autant «devenir indigène $»^{11}$. Épouses, mères, hôtesses, tentées parfois par les soies et les textiles si facilement accessibles, restent quand même dans leurs jupes et leurs corsets.

Échapper à ces cadres sociaux et officiels, à ces logiques commerciales et esthétiques, et transgresser les codes d'habillement

The Illustrated London News, 28 mars $1931: 3$.

10 Cassell $1865: 12$.

11 Cambridge (Grande-Bretagne), Centre for South Asian Studies (CSAS), Ferrar Papers, 27 July 1976. 
exigés par les formes de sociabilité anglaises n'est guère facile. Il y a toujours des occasions privées où cela s'est fait : les fêtes de Noël ou d'anniversaires quand les "déguisements» sont bien vus, ou des circonstances exceptionnelles imposées par des climats difficiles. Les femmes qui ont rompu les règles, telle Fanny Parkes, restent des exceptions. Fille d'un officier de l'armée anglaise et femme d'un modeste fonctionnaire anglais, elle s'indianisa au cours de ses vingtquatre années passées en Inde, apprit l'ourdou et préféra les vêtements indiens aux européens ${ }^{12}$. Ces cas peuvent s'analyser comme des formes d'ethno-mascarade, pour accéder à des lieux interdits aux Européens ou passer inaperçu dans la société locale ${ }^{13}$. Au contraire, Annie Besant (1847-1933), Margaret Noble (1867-1911) ou Margaret Slade sont des femmes engagées dans l'espace public, dont les positions politiques sont relayées par la presse nationale et internationale. Leurs choix vestimentaires relèvent d'une analyse politique.

\section{Esthétiques militantes}

Annie Besant arrive en Inde en 1893, porteuse déjà d'une expérience politique : elle est une militante de la Fabian Society en compagnie de Sidney et Beatrice Webb, et de George Bernard Shaw et elle a acquis une certaine notoriété du fait de ses actions en faveur des droits des ouvrières et de la planification familiale. Ayant quitté l'église anglicane et adhéré au mouvement théosophique fondé en 1875 par la Russe Héléna Blavatsky (1831-1891), qui s'appuie sur les principes hindous de karma et de réincarnation, Besant s'engage en Inde dans la lutte pour l'acceptation de l'Inde comme dominion au sein de l'empire britannique. Alors qu'elle a exprimé longuement ses idées politiques, en contraste, elle ne nous livre guère d'informations sur ses préoccupations vestimentaires. Une grande partie de la littérature sur Besant, y compris ses propres œuvres, se concentre sur sa trajectoire politique et expose ses idées sur la civilisation

12 Parkes 1850.

13 Said 1979 : 3 ; mais, dans ce cas, pourquoi ne pas appliquer la même notion d'ethno-mascarade aux adoptions d'habits européens par les Indiennes au lieu de modernisation ou d'européanisation? Cette idée est développée à partir d'un examen des pratiques vestimentaires des femmes européennes dans l'empire Ottoman et des femmes ottomanes en Europe par Konuk 2004 : 393-414. 
et la culture indiennes. Ils ne fournissent aucun élément sur sa vie sociale et ses sentiments. Quant à son autobiographie, elle s'arrête en 1893, au moment de son départ pour l'Inde.

Cependant, quelques photographies révèlent une transformation de son mode vestimentaire au fil du séjour indien. Les premières photos à son arrivée en Inde en 1893 la montrent en chemisier de dentelle, avec un col montant, recouvert d'un long tissu drapé très serré autour du corps (figure 1). Ses cheveux courts et son élégance vestimentaire lui donnent dans ce portait l'apparence d'une Anglaise issue de milieux aisés. Deux ans plus tard, le changement est frappant. Elle porte le même style de chemisier mais avec un large châle dont une partie tombe derrière son épaule gauche et l'autre est suspendue devant, selon le style adopté par certains hommes indiens de l'époque. Cette tenue correspond aux exigences de l'épouse traditionnelle de la classe moyenne de garder couverte la totalité du corps et de ne pas porter de vêtements trop voyants.

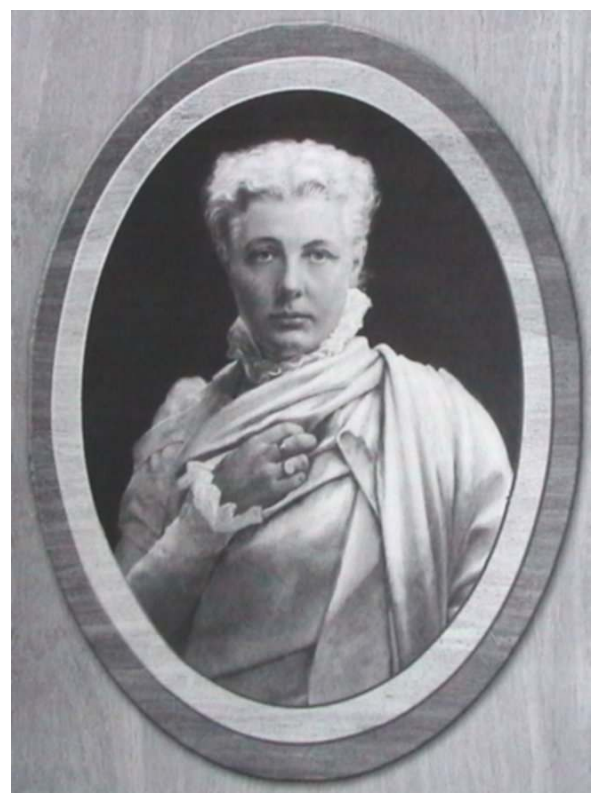

Figure 1. Annie Besant dans les années 1890. 
Des photos des années 1920 et 1930 montrent en revanche Annie Besant dans un vêtement drapé qui ressemble à un sari, couvert d'un châle. Sans doute, ces habits donnaient-ils une cohérence à son engagement dans la défense de la civilisation indienne, de l'hindouisme et de l'ancienneté du sanskrit : elle a appris le pali, visité les principaux sites hindous et déclare que son cœur est indien. Le sari est simple, avec une bordure, mais sans ornementation ni décoration (figure 2). Son drapé est ample, mais il est dépourvu de plis et ne donne aucun relief aux formes du corps, à la différence du drapé des Indiennes qui, serré à la taille, accentue fortement la poitrine, la taille et les hanches. Enfin, il s'apparente à une tunique, qui couvre tout le corps de haut en bas de façon uniforme.

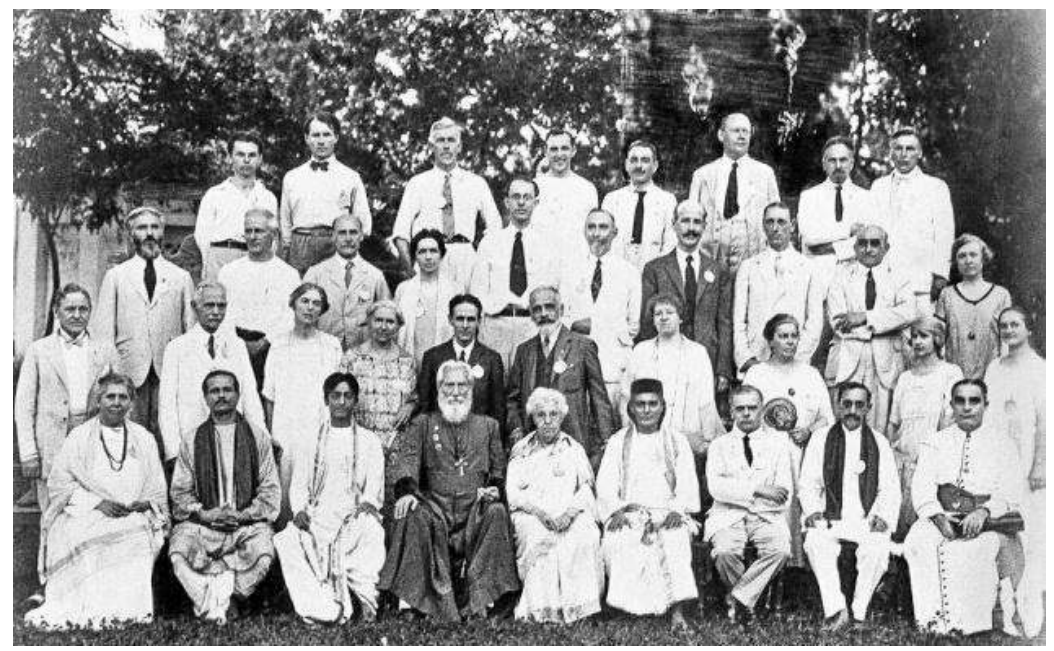

Figure 2. Annie Besant (au centre) au Jubilé d'Or de la Société Théosophique à Madras (1925).

C'est cette forme d'appropriation de l'un des éléments essentiels du vêtement féminin indien que je désigne sous l'expression de «saris européanisés ", expression qui n'appartient pas au vocabulaire des femmes de l'époque. S'il n'y avait pas sa coiffure - les cheveux courts, que ne portent jamais les Indiennes mais qui témoignent, en Europe, de l'engagement politique de quelques rares femmes, constituent 
paradoxalement l'élément peut-être le plus féminin de son apparence ${ }^{14}-$, Besant pourrait ressembler aux nationalistes indiens qui, dans le Sud et dans l'Ouest, portent eux aussi des vêtements drapés de type « dhoti $\rangle^{15}$.

Ainsi, elle réunit dans sa personne un symbole fort de l'indianité, à savoir le costume, qui interpelle forcément le public nationaliste auquel s'adresse son discours, et des éléments clairement européens, principalement les cheveux courts. Parce que les cheveux longs sont le signe à la fois public et physique qui définit le genre féminin en opposition à l'homme, les cheveux courts d'A. Besant la situent sans ambigüité du côté de la culture européenne. Puisque le port des cheveux courts est encore une arme nouvelle des Européennes désireuses de prendre leurs distances par rapport aux modes anciennes de la féminité éternelle et de la moralité publique, elle place Annie Besant dans un mouvement social féminin européen. En contraste, pour un œil indien, les cheveux courts de Besant expriment son étrangeté dans une société où la charge et la signification symbolique et idéologique des cheveux de femme sont encore trop fortes pour permettre des altérations ou des effets de mode personnelle ${ }^{16}$. Elle n'a jamais fait partie de la communauté des "memsabibs»-féminin de "sahib", désignant le colonisateur doté d'un statut supérieur-, «daughters of Britannia» ou «women of the Raj» ${ }^{17}$. Et elle n'a rien à faire avec ces groupes en Inde. Ses interlocuteurs sont principalement des hommes indiens, savants brahmanes ou personnalités politiques, bien qu'elle soutienne des causes sociales plus larges, telle l'éducation féminine après 1904. Le vêtement simple de Besant est en harmonie avec l'accent mis sur le boycott des produits anglais depuis le projet britannique de partition du Bengale en 1905. Gandhi, après son retour au pays en 1915, a effectué des changements radicaux dans son habit

14 Zdatny 1996 ; Synnott 1987.

15 Vêtement masculin, drapé autour de la taille, fait d'une unique pièce de tissu, le plus souvent de coton.

16 Les cheveux sont à la fois des signes de deuil ou de sacrifice et un symbole de dévouement au dieu dans l'hindouisme, le bouddhisme et le jainisme. Seules les veuves ou les moines se font raser. Le sujet reste négligé par les anthropologues et sociologues indiens. Une exception est l'étude des hindous punjabis par Hershman 1974.

17 Cf. Hickman 2000 ; Macmillan 1988. 
pour rechercher un signe d'indianité plutôt que d'appartenance à une communauté, une religion ou une région: il a épuré son style vestimentaire jusqu'à adopter un simple «dhoti», et a même abandonné le turban, pourtant signe traditionnel de l'autorité et du respect chez l'homme. Il est suivi par nombre de ses compagnons et disciples qui partagent le même souci : Motilal Nehru, avocat d'Allahabad, converti aux idées nationalistes après la Première Guerre mondiale, quitte ses habits occidentaux pour se vêtir en khadi, tissu de coton fabriqué artisanalement en Inde et ainsi symbole de lutte et d'identité indienne ${ }^{18}$.

À la même époque, dans l'Est de l'Inde, Margaret Noble, une Irlandaise, a aussi rejeté les habits européens. À première vue, elle semble être une intruse dans cette catégorie de femmes ayant adopté des "saris européanisés», car elle porte non pas un sari mais une longue robe, plus proche de celles des moines que de celles des femmes. Sa proximité avec l'ordre fondé par Vivekananda (né Narendranath Datta, 1863-1902) ${ }^{19}$ est peut-être à l'origine de ce choix (figure 3). Inspirée par ce maitre spirituel nationaliste qui a fait connaitre l'hindouisme en Occident, elle l'accompagne en Inde en 1898. Elle suit d'abord la mode victorienne, portant une longue jupe et un chemisier. Mais comme Vivekananda, qui, lors du Parlement des religions à Chicago en 1893, a fait de son turban et de ses robes orange une marque spectaculaire d'identité en Occident (figure 4), Noble entreprend aussi des modifications drastiques dans sa garderobe. Ces décisions sont prises au fur et à mesure de son assimilation dans la société indienne. D’une part, ses intérêts intellectuels la conduisent à côtoyer les élites bengalis du Brahmo Samaj, mouvement de réforme sociale de l'hindouisme et de la société hindoue, fondé dans les années 1830. D’autre part, elle est désireuse de s'assimiler aux milieux les plus modestes.

18 Bayly 1986 ; Bean 1989 ; Cohn 1996 ; Tarlo 1991, 1996, 2005 ; Ramagundam 2008.

19 Après son retour du Parlement des religions à Chicago en 1893, il fonde la Ramakrishna Mission, organisation vouée au travail social. Elle existe toujours avec plusieurs branches à travers le monde. 


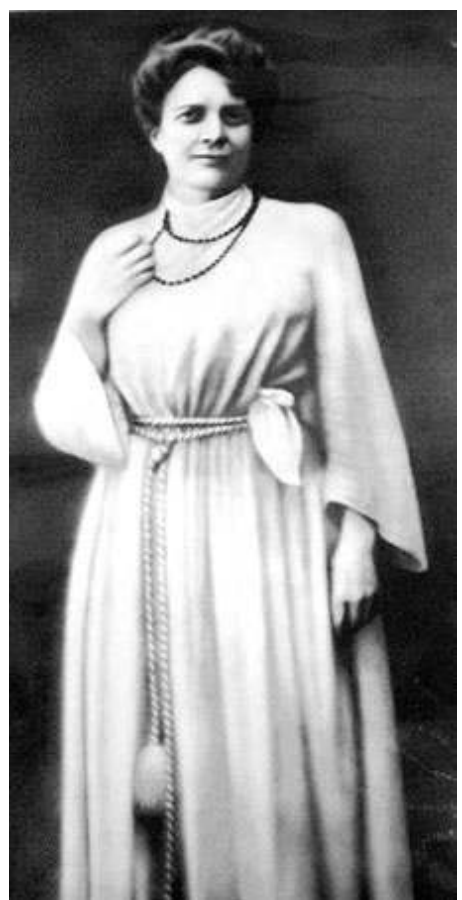

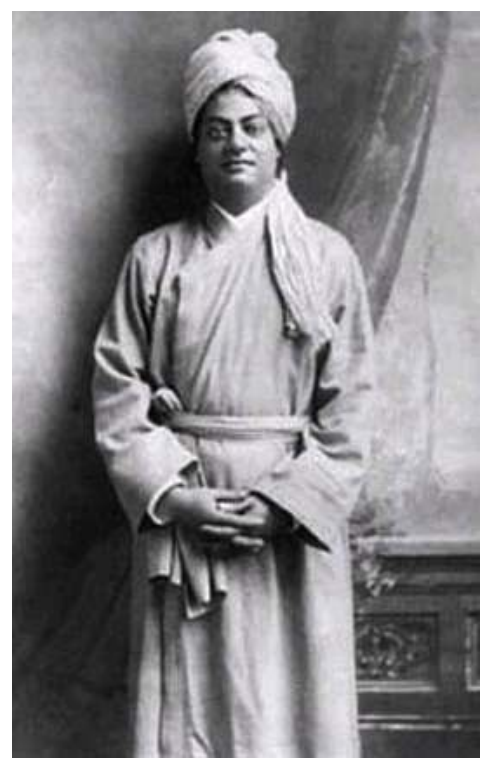

Figures 3 et 4. Margaret Noble, devenue Nivedita, 1906 (à gauche); Vivekananda, vers 1900 (à droite).

Ainsi, dès son arrivée, elle insiste et réussit à s'installer chez la femme du maitre spirituel de Vivekananda, Sarada Devi. Elle y mène une vie très simple et pauvre, suivant les pratiques spirituelles des femmes bengalis hindoues, veuves ou engagées dans une voie spirituelle, aux marges de la société. Quand elle s'installe dans un quartier pauvre de Calcutta, elle a quitté ses robes victoriennes pour une robe simple à la mode de son maitre spirituel. Elle n'explique pas ce choix qui semble répondre au contexte matériel modeste des quartiers hindous et à ses propres affinités qu'elle exprime dans ses écrits. Il est certain qu'il correspond à son engagement vis-à-vis des traditions philosophiques et religieuses hindoues et de leurs symboles. Elle évoquera ailleurs l'ancienneté et la perfection du sari :

$\mathrm{Au}$ début peut-être nous avons l'impression que le sari est étroit au niveau des chevilles, que sa forme ovoïde ressemble à un tas... Mais cela même est beau, et il y a une variété qui nous échappait d'abord. Il n'est 
pas trop serré, et au temps de loisir il donne aux pieds toute la liberté de la jupe. Et sa couleur !... Je languissais pour des saris sans bordures. Mais j'ai appris à l'apprécier. Cette petite ligne, qui semblait une interruption inutile dans une composition, me semble aujourd'hui comme le coup de pinceau d'un grand maitre, définissant et mettant le tout en valeur ${ }^{20}$.

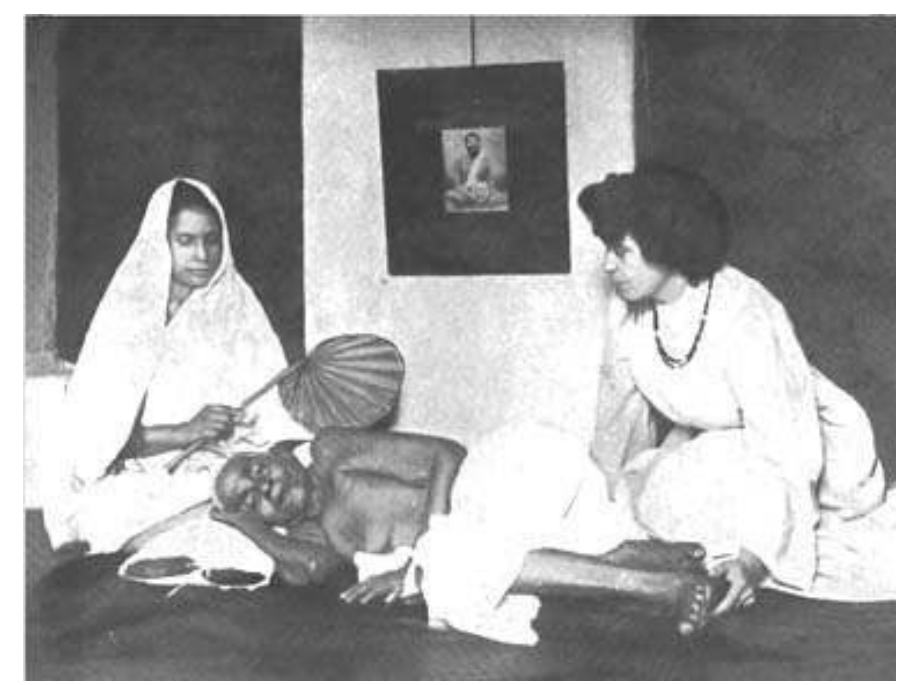

Figure 5. Margaret Noble (Nivedita) aux côtés de Gopala-Ma, disciple de Ramakrishna (1906).

Ces notes révèlent les préjugés de la femme occidentale sur le sari qui apparait à première vue tout à l'opposé de la notion européenne de costume féminin: vêtement coupé, cousu et modelé au corps d'une manière très travaillée. Le sari au contraire, parait étroit, sans forme ni style et contraignant un mouvement libre. Mais un examen plus proche révèle ses qualités esthétiques et non-contraignantes.

La robe de Margaret Noble, aux manches longues et larges, interrompue par une simple corde tressée, ressemble plutôt au froc des moines (figures 5). Abanindranath Tagore, le fils du poète et peintre lui-même, décrit sa longue robe blanche (en flanelle ou laine légère) et le chapelet fait de grains de rudraksha, ses cheveux bruns réunis en un

20 Noble $1913: 28-29$. 
chignon. La robe était pratique, une variante de la robe européenne, sans accentuation de la taille, sans déformation, avec des manches longues et larges ${ }^{21}$. Elle acquiert alors le statut de "Naisthika Brahmacharini » (célibataire), prend le nom de Nivedita (dévouée), mais garde la liberté d'une sœur laïque. Est-ce pour faciliter son acceptation et diminuer son statut de "sale étrangère » dont le contact physique polluerait? Sa coiffure - cheveux courts comme Besant - est le seul élément de continuité avec son passé et sa culture d'origine. Même avec cette concession à la vanité, son habillement est reconnu comme signe de renoncement selon les traditions sociales et spirituelles hindoues. Il donne force à ses prises de positions favorables à l'unité et la liberté de l'Inde, après la mort de Vivekananda en 1902 et lors de ses tournées de conférences dans l'Ouest et le Sud de l'Inde.

En effet, la reconnaissance publique des deux femmes est complète. Élue présidente de l'Indian National Congress en 1917, Besant joue un rôle de premier plan, entre les extrémistes et les modérés du mouvement nationaliste. Et si le travail de Nivedita est interrompu par sa mort précoce en 1911, elle s'inscrit néanmoins avec Besant dans le panthéon des Indiens nationalistes, statut confirmé par la production philatélique.

\section{Une politique du vêtement}

Le nouveau régime vestimentaire d'Annie Besant et de Nivedita s'inscrit dans le climat d'après-guerre en Europe et d'essor nationaliste en Inde. En Europe, les idées de confort corporel et l'accent mis sur les effets positifs de l'air et du soleil invitent à rendre moins contraignants les vêtements féminins. Le souci du bien-être physique commence à l'emporter sur une préoccupation exclusivement tournée vers l'apparence. Besant et Nivedita agissent dans ce contexte. En Inde aussi, hommes et femmes adaptent leurs vêtements pour mieux refléter leurs programmes et idées politiques ou correspondre à leurs nouveaux statuts et positions. L'émergence des mouvements nationalistes conduit leurs militants à des prises de position sur la question de l'habillement et à un débat sur la nécessité d'un habit

21 Foxe $1975: 164$. 
national dans les années 1920. Le refus de la soumission politique s'exprime par le rejet de modes occidentalisées, pourtant avidement adoptées par la première génération d'Indiens éduqués dans des institutions anglaises. Gandhi, Motilal Nehru, Jawaharlal Nehru, Aurobindo, Tagore, ont tous effectué la transition des costumes européens aux habits indiens. Leur adoption de dhotis, de turbans, de vestes soutient les produits Swadeshi (indigènes) contre ceux Videshi (étrangers) et le tissu indien (khadi) contre le système économique impérial. L'habit devient un moyen de communication politique. Gandhi élabore son célèbre dhoti en plusieurs étapes, à la recherche d'un vêtement le plus «national » possible, qui ne soit identifié ni à une région ni à un groupe particulier. Parfois, des mélanges hybrides entre modes indienne et occidentale aboutissent à des combinaisons telles qu'un sarong porté sur le pantalon à Ceylan ${ }^{22}$. Tandis que les hommes prennent leurs distances avec les costumes occidentaux et reviennent à des habits plus traditionnels (pyjama et tunique avec châle ou veste et turban), les femmes entreprennent également des modifications de leur tenue.

Leur accès progressif à l'éducation supérieure et leur envie de participer à la sphère publique les poussent à adapter leur costume. Les femmes des classes supérieures occidentalisent leur sari en le drapant sur une longue jupe, introduisant un chemisier aux manches longues. Ces styles sont d'inspiration occidentale, incluant une veste, des chaussures fermées et une broche à l'épaule pour tenir les plis du sari en place. Ils sont en rupture avec l'ancien système drapant le corps dans une seule longue pièce de tissu, sans chemisier. Les modes victoriennes, en conservant la pudeur, permettent aux femmes des classes supérieures de sortir en public ${ }^{23}$. Les premières Indiennes qui poursuivent leurs études à l'étranger comme Cornelia Sorabji, Pandita Ramabai ou Sarojini Naidu, s'habillent dans ces saris occidentalisés, tout en accentuant l'ornementation et la féminité de l'habit par d'autres marqueurs (bijoux, fleurs dans les cheveux, usage de vermillon sur le front ou dans la raie des cheveux...) qui indiquent leur statut (mariée, célibataire ou veuve). Le sari européanisé est ainsi

22 Pieris 2007.

23 Le phénomène est étudié par Karlekar 2006 : 2. 
une réponse aussi bien des Indiennes aux changements introduits par l'intervention coloniale que des Européennes désireuses d'agir sur la place publique selon les normes acceptées par la société indienne. Même si les changements d'habit ne font pas d'elles des Indiennes, car elles restent visiblement étrangères, elles se rapprochent d'elles.

Parmi ces femmes, seule Margaret Slade, alias Mirabehn, nous a laissé des informations précises sur son adoption du sari (figure 6). Dans son autobiographie, elle raconte sa décision de distribuer ses robes parisiennes chic avant de quitter l'Angleterre pour rejoindre l'ashram de Gandhi en Inde ${ }^{24}$. En même temps, elle devient végétarienne et abandonne l'alcool. Elle est déterminée à adopter le sari pour mieux s'intégrer dans la vie austère de l'ashram. Elle reçoit son premier sari en khadi d'Anusuya Behn, une femme habitant l'ashram. Sa transformation trouble Gandhi, mais il cède devant sa détermination. «Le plaisir que j’ai ressenti était gâché » dira-t-elle plus tard ${ }^{25}$.

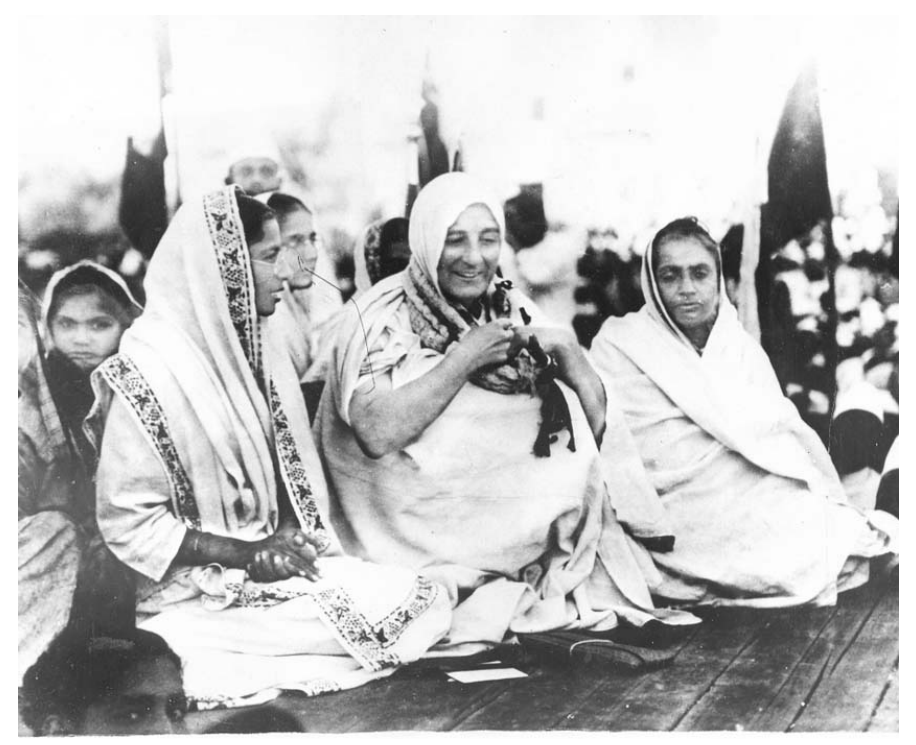

Figure 6. Mirabehn à Karachi (1931).

24 Slade $1960:$ 61-62.

25 Ibid. : 79-80. 


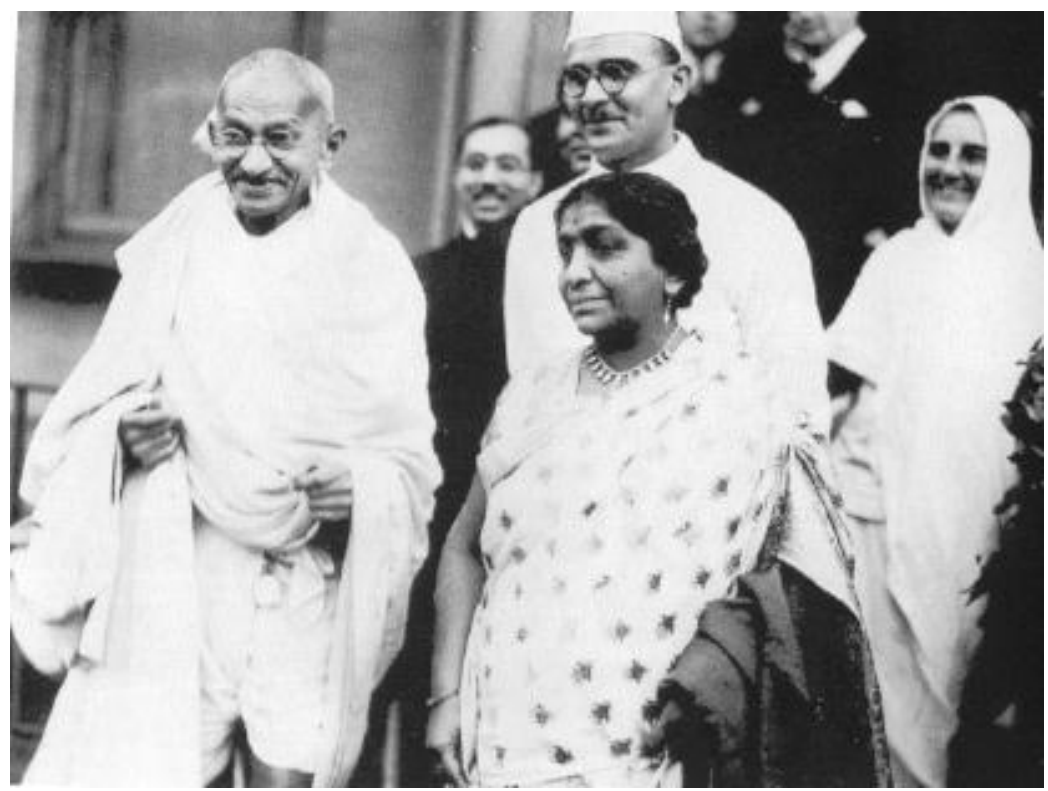

Figure 7. Gandhi, Sarojini Naidu et Mirabehn à Londres en 1931.

Gandhi a-t-il senti que cette transformation n'était pas convaincante pour les Indiens? Connaissant la tendance de ses compatriotes à prendre de haut toute imitation ou, au mieux, à la tolérer avec condescendance, il a dû juger ce geste trop provocateur pour les conventions sociales, problématique aussi bien pour les Indiens que pour les étrangers. En tout cas, il insiste pour qu'elle ne s'habille qu'en saris de khadi blanc pur sans bordure. Elle se confond ainsi avec d'autres groupes d'Indiennes qui affichent par là leurs sentiments patriotiques et leur engagement politique.

Crâne rasé, sari blanc, Mira s'inscrit aussi bien dans la tradition de Gandhi, qui mêle ascétismes jain et hindou, que dans la politique nationaliste gandhienne de simplicité et de minimalisme. Elle garde ces vêtements même durant ses voyages en Europe, par exemple aux côtés de Gandhi à Londres en 1931 (figure 7), puis lors de ses tournées en Angleterre comme porte-parole de Gandhi, indifférente aux 
commentaires qui la comparent, entre autres, à « un cowboy $»^{26}$. « Elle a rasé sa tête à la mode monastique... va nu-pieds, a brûlé tous ses vêtements européens, ne porte que les habits hindous les moins chers ». Elle a pris le nom d'une sainte mystique du Rajasthan, Mira Bai, figure centrale du mouvement des dévôts de Vishnou (Vaishnava bhak:ti) qui, née princesse, s'était vouée au dieu Krishna.

Un Européen nous livre le regard d'un occidental :

Qui est-elle, je me suis demandé, et puis la réponse est venue aussitôt ; elle doit être une femme hindoue disciple... dès qu'elle a parlé, je me suis rendu compte qu'elle était la femme anglaise dont tout le monde parlait. Son discours et sa manière directe me l'ont fait associer avec quelque chose hors du monde hindou. Elle était pieds nus, une figure solide dans une jupe et chemise en calicot, avec une veste sans manche, tissé à la main, qu'elle portait les soirs. La structure en proportions fines de son corps était saisissante. Elle avait le physique et la posture d'un cowboy. Elle me rappelait aussi certaines de nos femmes paysannes. Sa marche n'avait rien de la légèreté voletante de l'hindoue... Sur son crâne rasé, elle porte un voile en calicot, entourant son visage. Ceci, plus qu'autre chose, lui donne l'air d'un paysan anatolien. Elle s'assit au bord d'un matelas, un peu maladroitement je trouvais ${ }^{27}$.

Ce portrait révèle que, malgré son apparence hindoue, ses paroles et sa « manière directe » trahissent ses origines « en dehors du monde hindou ». La marche, le mouvement corporel ne suivent pas l'habillement.

Cette impression se renforce si on la compare aux Indiennes comme Sarojini Naidu, une militante nationaliste qui mettait un point d'honneur à s'habiller de saris de soie fine ou de coton. Même dans un sari très simple quoiqu'accompagné de boucles d'oreille et d'un collier, elle offre une apparence de féminité qui contraste avec l'ascétisme et la sobriété de Mirabehn. Il serait en effet parfois difficile d'identifier Mirabehn comme femme si nous ne connaissions pas déjà son genre (figure 7). Sa tête est couverte, son sari l'enveloppe. Au milieu d'Indiennes, elle a un air masculin qui suscite le trouble, chez les Indiens comme chez les Occidentaux. Dès lors, le sari renvoie plus à l'indianité qu'au genre.

26 Les journaux anglais adoptent unanimement cette interprétation lors de cette visite: The Evening Independent, 24 février 1931; The Telegraph, 24 février 1931 ; Daily Chronicle, 24 février 1931.

27 Edib $1937:$ 66-68. 
Dans les trois cas, l'habillement choisi n'est pas une simple imitation des modes indiennes d'une région spécifique. Il est à chaque fois personnel, plus simple que le style des Indiennes des élites, sans pli, sans ces jeux de draperie qui permettent de cacher et de révéler des parties du corps pour s'embellir et séduire. L'indianisation est modérée par les coupes de cheveux. Si Mirabehn, crâne rasé et vêtue de blanc, se rapproche des veuves hindoues ou des femmes jaines vouées à la voie spirituelle, Besant et Noble, en gardant leurs coiffures originales, envoient aux Indiens des signes d'occidentalité, voire de modernité.

Néanmoins, ces signes de leur identité britannique ne sont pas des obstacles à leur reconnaissance. La popularité d'Annie Besant dans le Sud est établie dès 1898, quand elle fonde le Central Hindu College à Benarès, et confirmée après son emprisonnement par le gouvernement anglais en 1916-1917 pour sa participation au Home Rule. Elle préside en 1917 la séance annuelle de l'Indian National Congress. Seules les critiques de certains Indiens expriment la défense des traditions orthodoxes hindoues, dans une société et une religion dominées par la pureté et les appartenances familiale et communautaire. L'assimilation d'Annie Besant est encore plus frappante dans le Sud, bastion de l'orthodoxie. Comme le note un récent commentaire :

Avec son entrée progressive dans la vie intérieure de notre pays, son apparence extérieure, sa routine quotidienne, et son mode de vie prirent une couleur indienne et elle est devenue une Indienne de part en part, une vraie hindoue en pensée, sentiment et action. Elle trouva l'âme de l'Inde par une communion interne ${ }^{28}$.

Et Nivedita est décrite comme « la dame anglaise gérant une école dans les quartiers indigènes... qui a adopté la mode indienne et s'appelle Sœur Nivedita ${ }^{29}$, bénéficiant de la reconnaissance des Indiens. Le chef de l'ordre des prêtres hindous à Bodh Gaya lui envoie un cadeau symbolique, qui représente la foudre; Aurobindo Ghosh, militant et partisan des actions révolutionnaires, est un de ses proches et le poète tamoul Subramanian Bharati reconnaît sa contribution à la diffusion des idées nationalistes.

28 The Hindu, 2 octobre 2003.

29 Foxe $1975: 210$. 
La réception de Madeleine Slade fut plus compliquée. Égale des autres femmes dans l'ashram, elle n'en restait pas moins la disciple anglaise de Gandhi, une curiosité pour les Anglais aussi bien que pour les Indiens. Cette position ne diminua pas le respect des hauts fonctionnaires et hommes d'État anglais. Quand elle apporte sa nourriture à Gandhi lors des rencontres avec Lord Irwin en 1931: «Bapu m’a présenté et Lord Irwin, se levant, m'a serré la main très amicalement ». Après son arrestation pour avoir participé à une réunion pour promouvoir le khadi, elle est conduite au poste de police où le commissaire discute avec elle et se lève pour lui serrer la main quand elle part $^{30}$. Mais pour les Indiens, sa situation était plus ambiguë. Elle servait certes d'intermédiaire entre eux et les Britanniques, qui respectaient ses origines familiales. Mais le fait qu'elle ait accepté de servir Gandhi au quotidien la réduisait, aux yeux des Indiens habitués à un système social qui méprisait le travail manuel, à une position de servante. Ainsi, quand elle apporte la lettre de Gandhi en 1942 annonçant le mouvement politique du "Quit India », elle note la surprise de Nehru qui lui dit: "Que diable fais-tu ici ? "31 Cette intimité avec Gandhi lui confère une position plus subalterne que Besant et Nivedita, qui étaient libres de manœuvrer et de négocier dans les milieux nationalistes.

Cette possibilité de façonner, de façon créative, leur corps par l'habillement est propre aux femmes. Les Britanniques ont abandonné à la fin du XIXe siècle l'ancienne habitude des colons européens de prendre des habits indigènes ${ }^{32}$. Désormais même ceux qui sont favorables à l'indépendance suivent les conventions établies par le gouvernement de Londres, qui les rendent identifiables en tant que Britanniques, sans le moindre signe d'origine de classe ou de statut. A.O. Hume, radical anglais, fonctionnaire dans les années 1849-1894, s'engage dans la fondation du parti du Congrès; intéressé par la religion et la culture indiennes, théosophe comme Annie Besant, il ne dévie pas pour autant de son identité de fonctionnaire civil, quoique

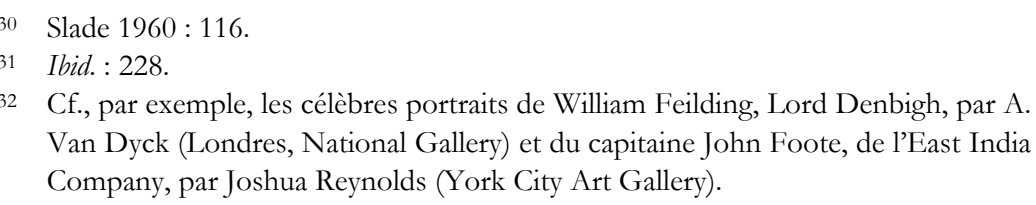


non conformiste. Même s'il se décrit en 1887 comme un natif de l'Inde ${ }^{33}$, il ne quitte pas pour autant ses habits anglais. Néanmoins, à partir des années 1920, quelques hommes manifestent plus de liberté. C.F. Andrews, ami de Gandhi et de Tagore qui, arrivé en Inde en 1904, milite pour la cause des dockers, des intouchables et des émigrants à Fiji, quitte ses habits occidentaux lorsqu'il est en compagnie d'Indiens. En résidence chez les Bose, un couple indo-américain, il adopte le «dhoti avec grande chemise, des sandales aux pieds, et marche nupieds dans la maison $»^{34}$. E.M. Forster, venu en Inde en 1921 comme secrétaire du prince hindou de Dewas, en Inde centrale, dévie parfois des normes impériales, pour participer aux rituels de la cour, dans l'intimité de la société Rajput. Mais il ne s'avoue pas très convaincu par ce changement. Il le décrit avec autodérision :

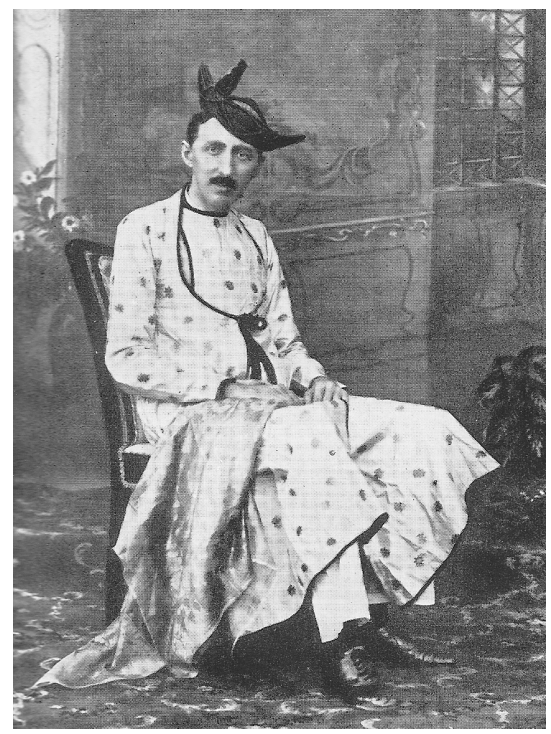

Figure 8. E.M. Forster à la cour du maharadjah de Dewas, 1921.
Aujourd'hui - anniversaire de l'empereur - il y avait un Durbar formel. J'ai porté une longue robe violette parsemée de fleurs dorées, sur une veste verte, elle aussi dorée, et sur ma tête une parure fantastique en rouge et or; également des pantalons blancs : la robe et la veste avaient des bordures rouges censées s'accorder avec le turban, ce qui n'était pas le cas. Je me trouvais complètement idiot (mais très admiré)... J'ai une garde-robe bien indienne maintenant ${ }^{35}$.

Pour lui, cela ressemble davantage à une mascarade.

33 Moulton 1985.

34 CSAS, Bose papers.

35 Forster 1953: 91. 
Comment comprendre ces choix vestimentaires? Bricolage, métissage, hybridation? À l'évidence, le costume de ces trois femmes est le produit d'une forme de bricolage, qui met ensemble des éléments hétérogènes, empruntés aux Anglais mais plus encore aux Indiens. S’il cherche à exprimer un changement de camp, il pourrait être interprété comme l'incapacité corporelle de femmes socialisées dans certains vêtements à passer à d'autres pratiques indissociables des vêtements indiens. Il peut plus encore apparaittre comme l'une de ces nombreuses opérations construites de métissage, au moment où s'opèrent des rencontres entre plusieurs cultures, mais un métissage inversé dans lequel les cultures dominées semblent s'imposer à celle des dominants. La question a été posée en termes d'hybridation par les travaux de Homi Bhaba, qui y voit un processus ouvrant un troisième espace d'énonciation pour les colonisés, séparé des espaces de significations originaires, et qui fonctionne comme un «lieu ambivalent où le sens culturel et la représentation n'ont aucune unité primordiale ou fixité ». ${ }^{36}$ Dès lors, ces «saris européanisés» ne peuvent plus être considérés comme des objets incomplets, ou "bâtards", des échecs qui témoigneraient de l'incapacité de ces femmes à changer de culture. Cette hybridation s'affirme davantage comme une construction forte permettant d'imposer une position au sein d'un champ hautement conflictuel.

Tout comme les nationalistes indiens, ces femmes n'adoptent pas un habit indien tel qu'il existe déjà, car cela aurait impliqué de porter un vêtement lié à une région, une communauté ou un statut social. Cela les aurait empêché de manifester leur adhésion à la nation indienne dans son ensemble. Ainsi, l'invention d'un vêtement proche d'un sari exprime sans ambiguité les positions politiques de ces femmes, et ces «saris européanisés » ne sont qu'un cas de figure dans un contexte plus large.

En élaborant ces habits, ces femmes affichent à la fois leurs différences et leurs affinités, une double distance, l'une évidente, visà-vis des élites anglaises dont elles sont issues, l'autre, plus subtile, à l'égard des élites indiennes. Celles-ci utilisent entre autres le sari comme un signe féminin de richesse et un marqueur de séparation

36 Bhabha $1994: 35$. 
vis-à-vis des couches sociales inférieures. En ce sens, le «sari européanisé » s'oppose à cela et introduit une exigence d'égalité sociale qui élargit leur protestation anticoloniale vers un programme plus social, avec des dimensions ouvertement féministes.

Enfin ces saris s'adressent à un public féminin. Comme l'ont noté des études sur la modernité des Indiennes, celles qui commencent à se montrer dans l'espace public adoptent des modes victoriennes dès la fin du XIXe siècle (chemisier avec col montant et manches longues, jupe sur laquelle est ensuite drapé le sari). Elles peuvent ainsi s'afficher aussi progressistes et modernes que le veulent leurs pères et maris, tout en satisfaisant leurs exigences patriarcales. Les exemples d'Annie Besant, de Margaret Noble (Nivedita) ou de Margaret Slade introduisent au contraire des aspirations à plus de liberté, d'individualité et de féminité ; le sari européanisé propose un style mieux adapté aux rôles publics et civiques, sa forme ample permet un mouvement facile et donne plus de liberté corporelle. Son usage fragilise l'un des consensus qui se sont construits entre colonisateurs et nationalistes. Au lieu de son usage traditionnel d'«identifiant sexué », le sari, dans une de ces formes hybrides que proposent Annie Besant, Nivedita ou Margaret Slade, peut devenir un «identifiant politique».

\section{Bibliographie}

BAYly Christopher A., 1986, "The origins of swadeshi: cloth and Indian society, 1700-1930", in Arjun Appadurai (ed.), The Social Life of Things: Commodities in Cultural Perspective, Cambridge, Cambridge University Press, p. 285-321.

BEAN Susan S., 1989, "Gandhi and Khadi: The Fabric of Independence », in Annette B. SCHNEIDER \& Jane WEINER (eds), Cloth and Human Experience, Washington, Smithsonian Institution Press.

BHABHA Homi K., 1994, The Location of Culture, Londres, Routledge.

Bhatia Nandi, 2003, "Fashioning women in colonial India », Fashion Theory, 7, p. 327-344.

BRADSHAw George, 1884, Through routes overland guide and handbook to India, Egypt, Turkey, Persia, China, Australia and New Zealand, Londres, W.J. Adams.

BUETTNER Elizabeth, 2004, Empire Families. Britons and Late Imperial India, Oxford, Oxford University Press. 
Cassell's emigrants' Handy guide to all the principal British colonies throughout the world [1865], Londres, Cassell, Petter \& Galpin.

CoHN Bernard S., 1996, "Cloth, clothes and colonialism: India and the 19th Century ", in Annette. B. SCHNEIDER \& Jane WeINER (eds), Cloth and Human Experience, Washington, Smithsonian Institution Press, p. 106-162.

Diver Maud, 1909, The Englishwoman in India, Edimbourg, W. Blackwood.

EDIB Halide Adivar, 1937, Inside India, Londres, Allen \& Unwin.

FoXe Barbara, 1975, Long Journey Home: A Biography of Margaret Noble, Londres, Rider.

FORSTER Edward M., 1953, The Hill of Devi, New York, Harcourt Brace.

GOPAL Sarvepalli \& Uma IYENGAR (eds), 2003, The Essential Writings of Jawaharlal Nehru, vol. 1, Delhi, Oxford University Press.

Hershman Paul, 1974, « Hair, Sex and Dirt », Man, 9, p. 274-298.

Hickman Katie, 2000, Daughters of Britannia: the lives and times of diplomatic wives, Londres, Ted Smart.

Indian Outits and Establishments. A practical guide for persons about to reside in India by an anglo Indian, 1882, Londres, Upcott Gill.

JOHNSON Donald C., 2007, "Clothes make the Empire: British dress in India », in Donald C. Johnson \& Helen B. Foster, Dress Sense. Emotional \& Sensory Experiences of the Body \& Clothes, New York, Berg.

KARLEKAR Malavika (ed.), 2006, Visualizing Indian Women 1875-1947, New Delhi, Oxford University Press, Women's Development Studies.

KonUK Kader, 2004, «Ethnomasquerade in Ottoman-European Encounters. Reenacting Lady Mary Wortley Montagu », Criticism, 3, p. 393-414.

LytTLETOn Katherine Neville, 1892, How to Pack, How to Dress, How to Keep Well on a Winter Tour in India, Londres, E. Stanford.

Macmillan Margaret, 1988, Women of the Raj, Londres, Thames \& Hudson.

Midgley Clare (ed.), 1995, Gender and Imperialism, Manchester, Manchester University Press.

Moulton Edward C., 1985, «Allan O. Hume and the Indian National Congress, a reassessment ", Journal of South Asian Studies, 8, p. 5-23.

NoBLE Margaret Elizabeth, 1913, Studies from an Eastern Home, Londres, Longmans, Green.

PARKES Fanny P., 1850, Wanderings of a Pilgrim, in search of the picturesque, during four-andtwenty years in the East, Londres, Pelham Richardson.

PIERIS Anoma, 2007, « The Trouser under the Cloth: Personal Space in ColonialModern Ceylon ", in Peter SCRIVER \& Vikramaditya PRAKASH (eds), Colonial 
Modernities. Building, dwelling and architecture in British India and Ceylon, Londres, Routledge, p. 199-218.

Ramagundam Rahul, 2008, Gandhi's Khadi: A History of Contention and Conciliation, Delhi, Orient Longman.

SAID Edward W., 1979, Orientalism, New York, Vintage Books.

SHRIMPTON J., 1992, «Dressing for a Tropical Climate: The role of Native Fabrics in

Fashionable Dress in Early Colonial India », Textile History, 23, p. 55-70.

SLADE Madeleine, 1960, The Spirit's Pilgrimage, Londres, Longmans.

STEel Flora Annie \& Grace Gardiner, 1890 [1888, 1re éd.], The Complete Indian Housekeeper and Cook giving the duties of mistress and servants, the general management of the bouse and practical recipes for cooking in all its branches, by two twenty years' residents, Edimbourg, F. Murray.

SYNNOTT Anthony, 1987, «Shame and Glory: a Sociology of Hair», The British Journal of Sociology, 3, p. 381-413.

TARLo Emma, 1991, "The Problem of what to wear: the Politics of Khadi in Late Colonial India », South Asia Research, 11/2, p. 134-157.

—, 1996, Clothing Matters: Dress and Identity in India, Londres-Chicago, University of Chicago Press.

—, 2005, «Khadi» (www.soas.ac.uk/southasianstudies/keywords/file24807.pdf ; consulté le 22 mars 2012)

Thomas Nicola J., 2007, «Embodying Imperial Spectacle: Dressing Lady Curzon, Vicereine of India 1899-1905 », Cultural Geographies, 14/3, p. 369-400.

Trollope Joanna, 1983, Britannia's daughters. Women of the British Empire, Londres, Pimlico.

ZDATNY Steven 1996, «La mode à la garçonne, 1900-1925 : une histoire sociale des coupes de cheveux », Le Mouvement Social, 174, p. 23-56. 